\title{
Application of Finite Element Sub-Modeling Techniques in Structural Mechanics
}

\author{
Evaldas NARVYDAS*, Nomeda PUODZIUNIENE**, Abdul Khan THORAPPA*** \\ *Kaunas University of Technology, Studentu st. 56, LT-51424 Kaunas, Lithuania, E-mail: evaldas.narvydas@ktu.lt \\ **Kaunas University of Technology Studentu st. 56, LT-51424 Kaunas, Lithuania, E-mail: nomeda.puodziuniene@ktu.lt \\ ***Kaunas University of Technology Studentu st. 56, LT-51424 Kaunas, Lithuania, E-mail: abdul.thorappa@ktu.edu \\ crossref http://dx.doi.org/10.5755/j02.mech.25962
}

\section{Introduction}

Initial discussion on displacement and force based sub-modeling techniques was presented in the conference Mechanika 2014 [1]. This paper extends the conference discussion adding case study of the nested sub-modeling approach for the maximum stress analysis in the weld toe of the surge vessel. Sub-modeling here assumes a finite element modeling technique employing two or more models of different geometry. The first model consists of a relatively coarse meshed geometry with possible significant errors of calculated results in some small regions. The second model (sub-model) contains a much close approximation of these regions, e.g. stress concentration region, but excludes the major part of the first model where results calculated by a coarse meshed model had acceptable accuracy. Visually it could be presented as a relatively small refined part (submodel) cut out from the global model. The boundary conditions of the cut-out edges or surfaces are based on the results calculated by the global model.

The cut boundary conditions of the sub-model often are determined by an interpolation of the calculated nodal displacements of the global model to the cut boundary nodes of the sub-model. In this case, the cut boundary displacements are valid only if the refinement of the sub-model does not change the stiffness of the sub-model region significantly comparing with the stiffness of the same region of the initial global model. It is a major drawback of this approach.

The mentioned drawback can be overcome using the force based cut boundary conditions of the sub-model, although purely force based boundary conditions will not restrict rigid body motion and additional displacement restraints should be applied. Therefore, the sub-model with the force based cut boundary conditions cannot be used for calculation of global displacements.

The sub-modeling approach is being used for decades in local stress analysis of large complex structures [2], contact zones [3], stress concentration zones [4] and crack tip zones $[4,5]$. Increasing power of the computational devices have not eliminated the sub-modeling approach from the engineering applications but replaced the initial relatively simple tasks of model reduction with the new challenging problems. In recent years, M. Perić et al. [6, 7] used sub-models for structural and thermal analysis of large welded panels. Sub-modeling technique was applied for structural integrity analysis of nuclear reactor pressure vessel $[8,9]$. This method allowed simplified 3D modeling of cracks at different locations and with arbitrary orientation. Sung et al. $[10,11]$ modeled crack initiation and fracture mechanics of pressure tube specimens; also curved compact tension specimens machined from irradiated pressured tubes employing sub-models to simulate fracture behavior ahead of the front of a crack.

Sub-modeling technique was used to refine the mesh required by the fracture analysis in the region of interest of postulated cracks [12] in modelling of reactor pressure vessel subjected to pressurized thermal shock. Brittle fracture assessment of field welded tanks for storage of production liquids was performed by modeling the cleanout junction as a three-dimensional sub-model, using solid elements, driven by a three-dimensional model of the entire tanks using shell elements [13].

Sub-models of the zones of stress concentration were included in shape optimization procedure [14] of the beater wheel. The riveted metallic bridge fatigue assessment [15] was performed by application of sub-modeling of the joint of interest by solid elements with boundary conditions transferred from the global beam type model.

J. Pilthammar et al. presented a sub-modelig technique in sheet metal forming simulation [16]. Application of the sub-modeling techniques for wear predictions is discussed in [17]. Analysis of the contact pressure and wear of hard bearing couples in hip prosthesis employing sub-modeling is recently published by S. Shankar et al. [18].

Contemporary sub-modeling procedures are supported not only by "classical" finite element codes (ABAQUS, ANSYS Mechanical APDL, NASTRAN etc.) but also appears in an integrated CAD/CAE software (SOLIDWORKS Simulation; ANSYS Workbench etc.). Commercial finite element code ABAQUS has procedures for "Node-based submodeling" and "Surface-based submodeling" [19]. Nodal-driven results of displacements, rotations, temperature, pore pressure and acoustic pressure are interpolated from the global model onto the cut boundary nodes of the sub-model in "Node-based submodeling". "Surface-based submodeling" procedure applies surface tractions to sub-model surfaces based on a stress field interpolated from the global model. ANSYS sub-modeling procedures map degree-of-freedom results (displacements) and body force loads (mainly temperatures for a structural analysis) from the coarse model to the cut boundaries of the submodel [20]. Sub-modeling procedure in SOLIDWORKS is highly automated. The disadvantage is that the part of the global model, intended to be analyzed as a sub-model, should be included in a global model as a separate component (part or body). This part should have bonded contact and incompatible mesh with the rest of the global model. However, it is still displacement based sub-modeling approach [21]. 
Procedure of the force based cut boundary submodeling was developed [1] for the ANSYS Mechanical APDL software employing FSUM command for nodal force extraction and CEINTF command to generate constraint equations at the cut boundary of the sub-model and to transfer the force action to the sub-model. The article demonstrates how displacement and force based sub-modeling techniques work in stress concentration zones of 2D solids. The influence of the mesh of the global model is investigated. Advantage of force based sub-model is discussed.

\section{Application of displacement and force based sub- modeling techniques}

The typical example of the sub-modeling application is a stress concentration problem in a plate with a hole under distributed tension load $p$ (Fig. 1). For comparison of the displacement and force based sub-modeling techniques, the square plate model was constructed. The 2D geometry and load parameters were as follows: $H=300 \mathrm{~mm}$; $2 a=30 \mathrm{~mm}$ and $p=100 \mathrm{MPa}$ (Fig. 1). Due to symmetry, a quarter of the geometry was employed for global finite element model with the proper boundary conditions. The submodel includes a segment of the plate with outer radius of $45 \mathrm{~mm}$ and angle $45^{\circ}$, as presented in Fig. 1, b.

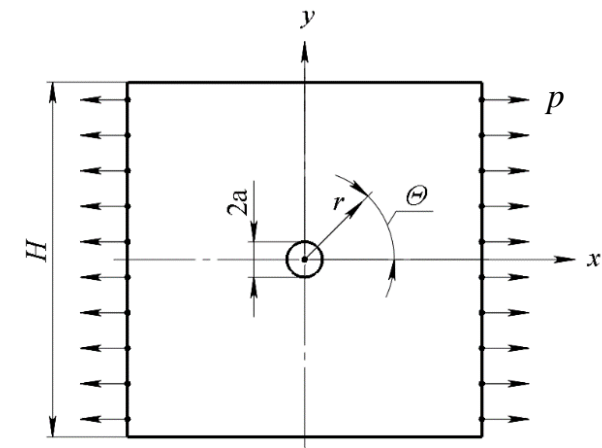

a

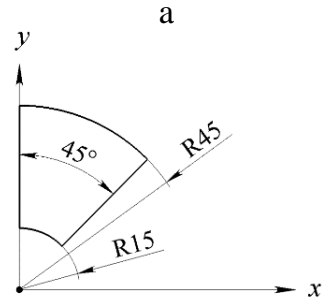

b

Fig. 1 Geometry of the plate with a hole (a) and a sub-model (b)

Analytical solution of the maximal elstic circumferential stresses in $2 \mathrm{D}$ polar coordinate system $\sigma_{\Theta \max }$ for the case depicted in Fig. 1 is known. It can be calculated emploing expression of elstic stress concentration factor $K_{t n}$ presented in Peterson's [22] (1), or Rork's [23] (2) hanbooks, where the first handbook uses works of Howland R. C. J., and the second is based on publications of Flynn P. D. and Heywood R. B.:

$$
\begin{aligned}
& K_{t n}=2+0.284\left(1-\frac{2 a}{H}\right)-0.6\left(1-\frac{2 a}{H}\right)^{2}+ \\
& +1.32\left(1-\frac{2 a}{H}\right)^{3},
\end{aligned}
$$

$$
\begin{aligned}
& K_{t n}=3-3.13\left(\frac{2 a}{H}\right)+3.66\left(\frac{2 a}{H}\right)^{2}-1.53\left(\frac{2 a}{H}\right)^{3}, \\
& \sigma_{\Theta \max }=K_{t n} \cdot \sigma_{\Theta n},
\end{aligned}
$$

here: $\sigma_{\Theta n}=p \cdot H /(H-2 a)$. Then, $\sigma_{\Theta \max }=303.5 \mathrm{MPa}$, if Eq. (1) is used and $\sigma_{\Theta \max }=302.5 \mathrm{MPa}$ if Eq. (2) employed.

The solution of highly refined plane stress global model gives results of $\sigma_{\Theta \max }=308.4 \mathrm{MPa}$. The coarse global models where the sub-modeled part (Fig. 2, d) had $3 \times 3,4 \times 4$ and 5x5 PLANE182 ANSYS finite element meshes (Fig. 2a - c) gave results of maximum stress 273, 289 and $298 \mathrm{MPa}$ (Fig. 3, a - c). The sub-models with displacement based cut boundary conditions of these global models resulted in $\sigma_{\Theta \max }: 306 ; 309$ and $311 \mathrm{MPa}$ respectively (Fig. 4, a-c). The relative difference comparing to fine global model solution is less than $1 \%$ and the difference comparing to solution according Eqs. (1) and (2) is less than $3 \%$.

The action of forces was transferred to the submodel by constraint equations connecting selected nodes of the global model and elements of the sub-model by specially written subroutine and CEINTF command. This have produced local peaks of stresses at the boundary (Fig. 5, a). However, excluding the elements with local peaks (selecting the region away from these elements as in Fig. 5, b) gives a proper character of the stress distribution in the submodel (Fig. 6). The maximal circumferential stresses $\left(\sigma_{\Theta \max }\right)$ for the same cases as in displacement based submodels are: 346.5, 330 and $321 \mathrm{MPa}$ (Fig. 6, a-c).

This allows to conclude that the mesh density of the global model is more important for the force based submodel than for the displacement base sub-model since only the $5 \times 5$ mesh of selected region of the global model gives an acceptable results with the relative error less than $5 \%$.

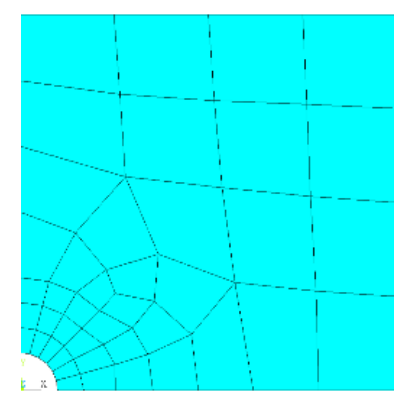

a

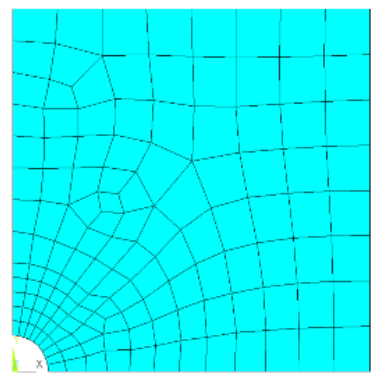

C

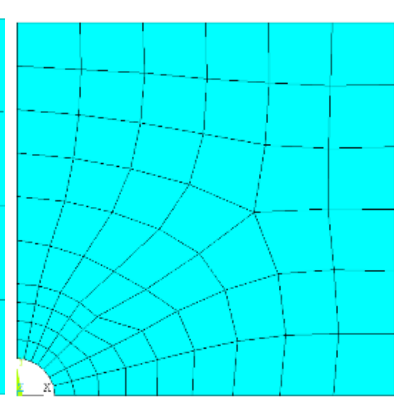

b

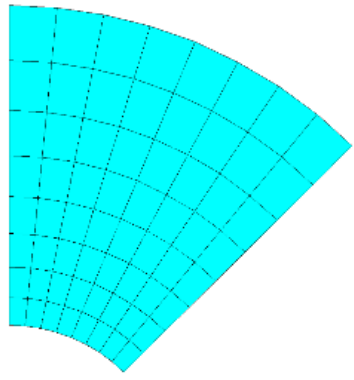

d
Fig. 2 Three cases of the tested mesh of general model (a, b and c) and the sub-model (d) 

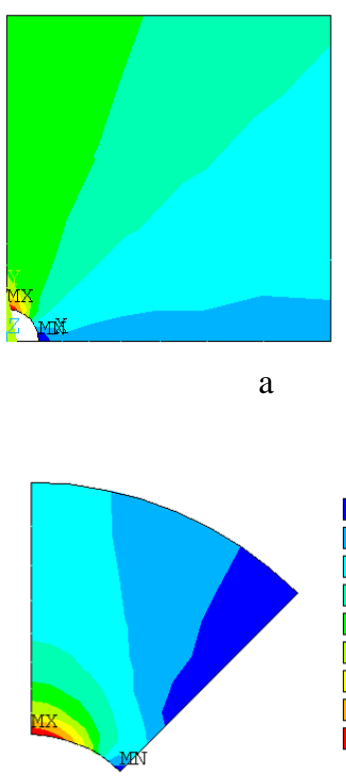

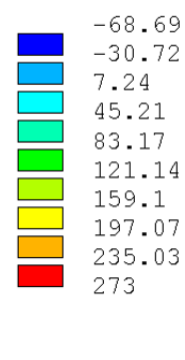

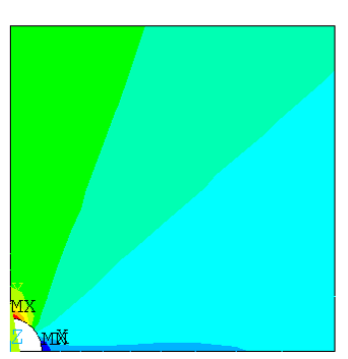

b

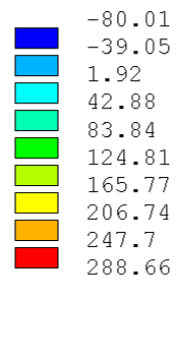

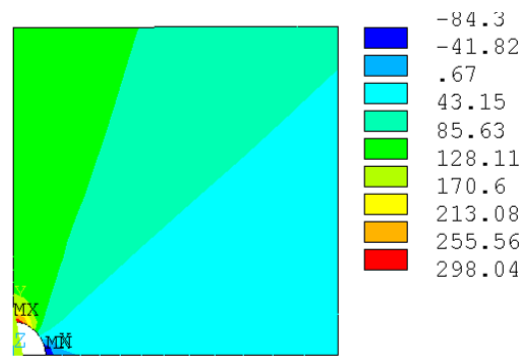

c

Fig. 3 Stress $\sigma_{\Theta}$ results using coarse meshes (a, b and c)

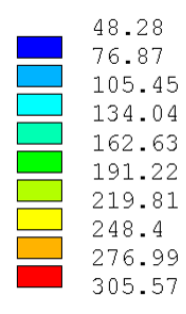

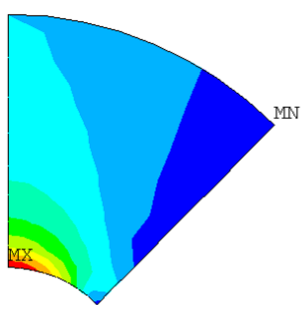

b

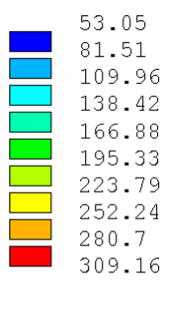

109.96

38.42

6.88

3.79

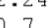

09.16

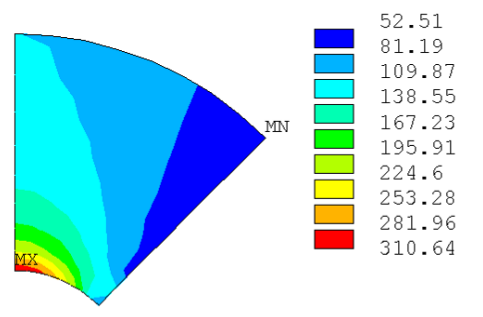

C

Fig. $4 \sigma_{\Theta}$ results of displacement based sub-model with different mesh of global model (a, b and c)

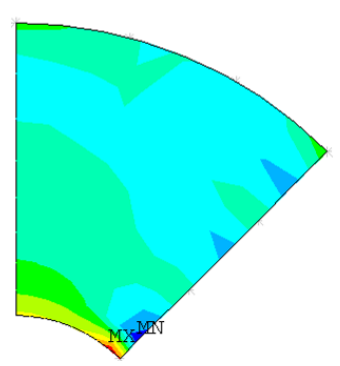

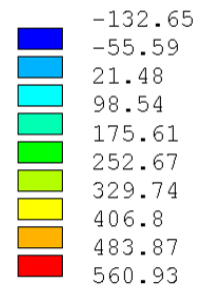

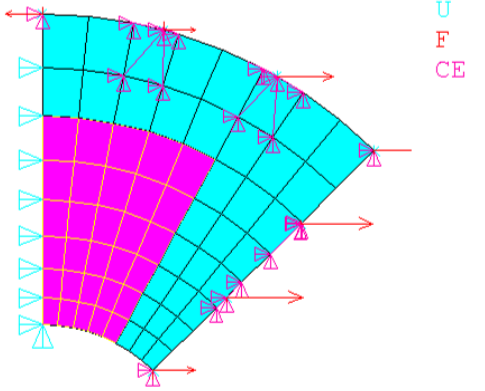

b

Fig. 5 Stress $\sigma \Theta$ peaks on the boundary of sub-model applying forces transferred from the global model (a) and selected region to avoid local stress peaks (b)

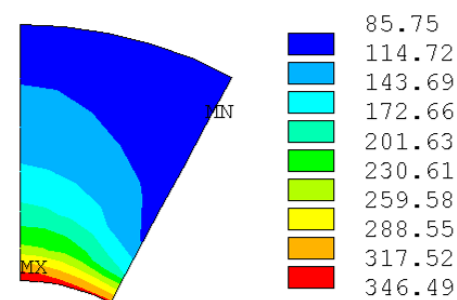

a

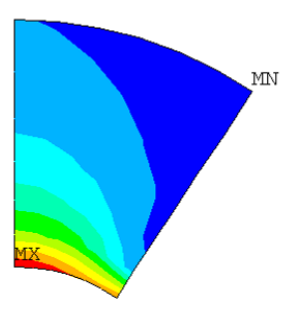

b

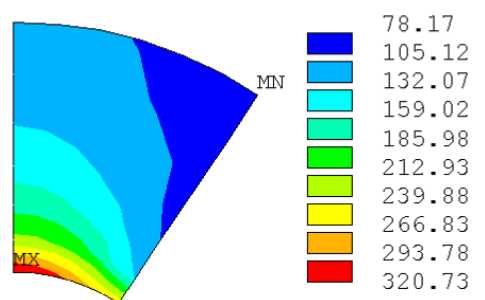

C

Fig. $6 \sigma_{\Theta}$ results of cut boundary force based sub-models under different meshes of global model (a, b and c)

The plate with the hole modelling results demonstrated that the displacement based sub-modelling has higher accuracy and is less sensitive to the mesh density of the global model. Therefore it should be used in most cases. However, force based sub-modelling has a privilege to maintain cases where stiffness of sub-model changes significantly e.g. changing the local geometry. Further it will be discussed by stress analysis of the component (Fig. 7, a) where the sub-model of the component (Fig. 7, b) has a different geometry comparing to the initial shape of the same region of the global model. Changing that geometry - a fillet radius from 10 to $80 \mathrm{~mm}$, the stiffness of the sub-model is changed. Three finite element models were used to illustrate stress difference in displacement and force based sub-models: the coarse meshed global model, sub-model with vary- ing step fillet radius, and refined global model for reference for the "exact" stress results (Fig. 8, a-d).

Fig. 9 shows equivalent (von Mises) stress results in displacement based sub-model (a), force based sub-model (b) and force based sub-model without boundary elements with local stress peaks (c), when fillet radius is $30 \mathrm{~mm}$ and load $100 \mathrm{MPa}$. Fig. 10 presents equivalent stress results in refined global model for comparison. The maximal equivalent stresses of displacement and force bases sub-models were normalized to the maximal reference stress. The results are presented in Fig. 11. These results demonstrate increasing deviation from the exact solution represented by normalized value 1 of stresses of the displacement based sub-model as the fillet radius $R$ is increasing. 


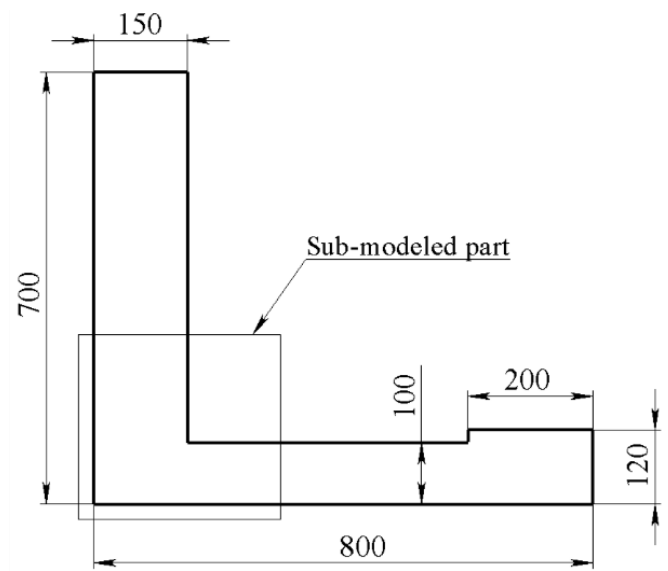

a

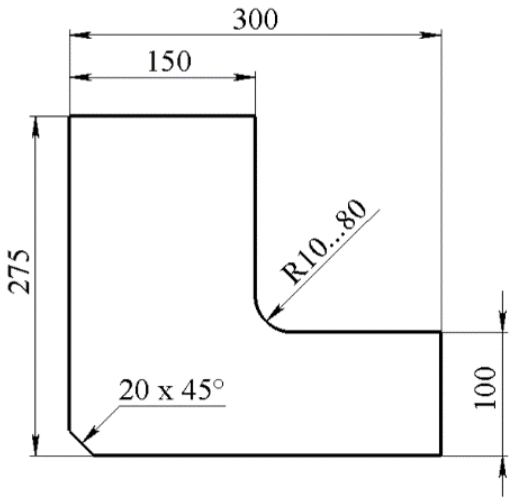

b

Fig. 7 Component geometry for global model (a) and a sub-model (b)

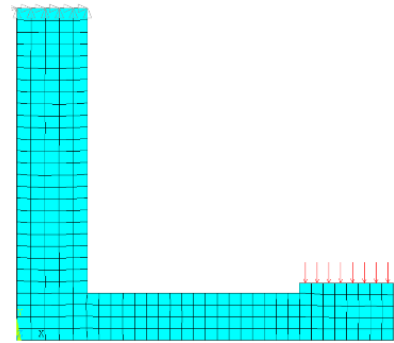

a

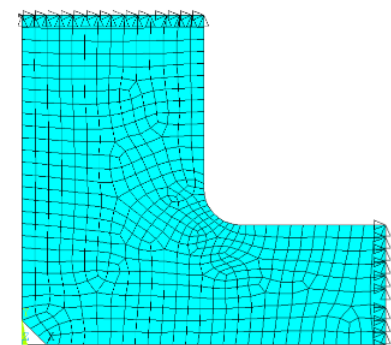

b

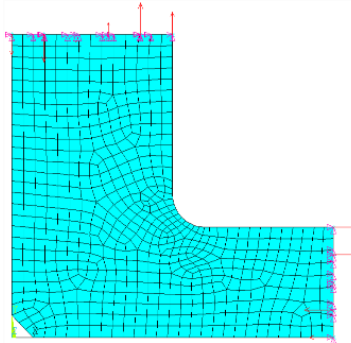

c

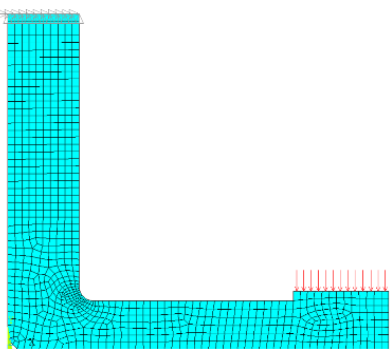

d

Fig. 8 Finite element meshes and loads of global model (a) displacement and force based sub-models (b and c), and refined global model for comparison $(\mathrm{d})$

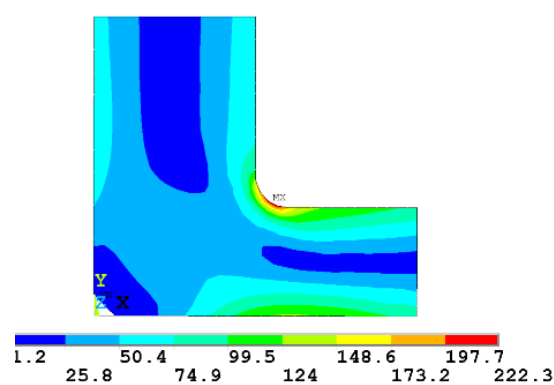

a

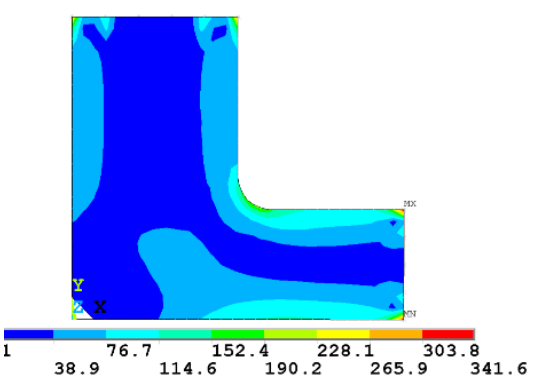

b

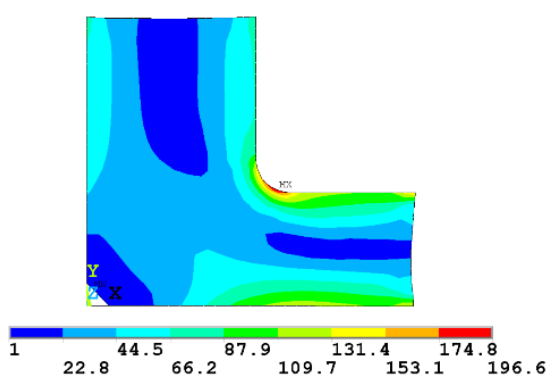

c

Fig. 9 Equivalent (von Mises) stress results in displacement based sub-model (a), force based sub-model (b) and force based sub-model without boundary elements with local stress peaks (c); fillet radius $30 \mathrm{~mm}$

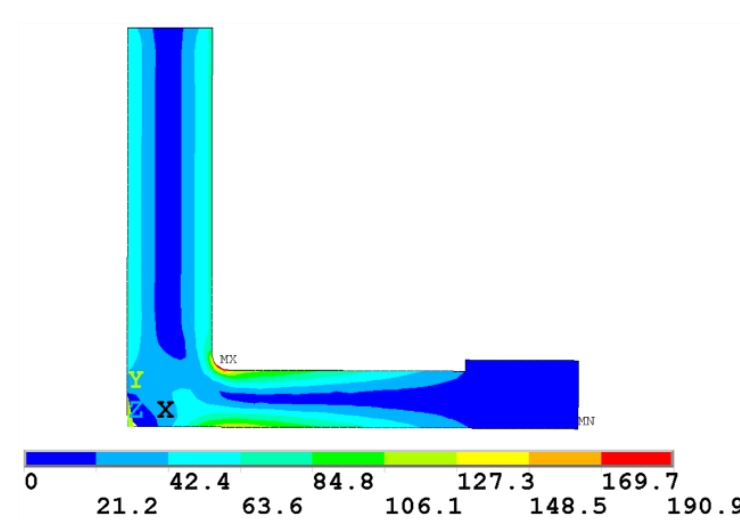

Fig. 10 Equivalent (von Mises) stress results in refined model for comparison

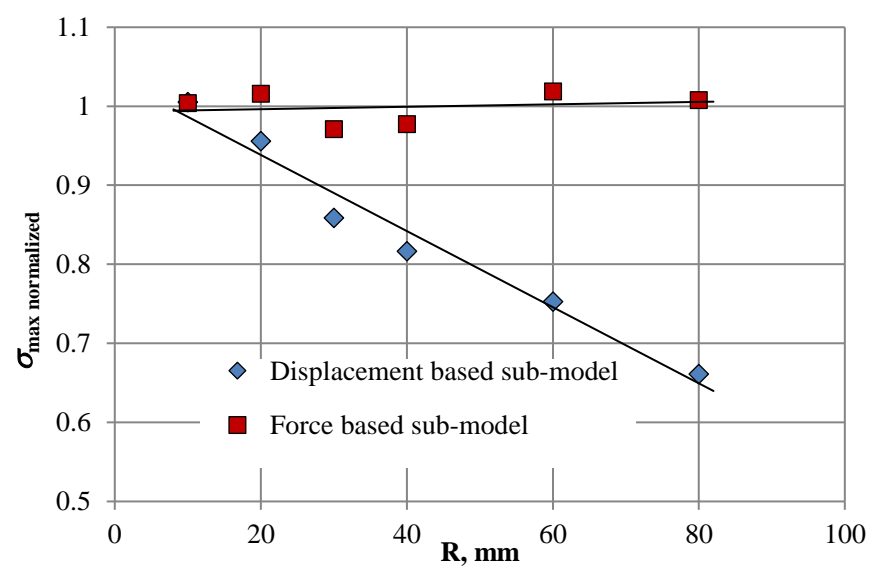

Fig. 11 Normalized maximal equivalent stress results in displacement and force based sub-models under varying fillet radius 


\section{Nested sub-modeling of surge tank weld toe}

The nested sub-modeling technique is called when the sub-model for the region of interest is applied more than once. For the case of interest, the global model of surge tank has a complicated structure (Fig. 12) and a finite element stress analysis of the entire tank cannot be done with the needed accuracy. Application of the sub-modeling technique is an appropriate solution used in similar cases [13]. However, the definition of the sub-model geometry often is not clear and the initial assumption could be not the best for the sub-modeling. Fortunately, the nested sub-modeling technique can be applied, where the initially selected submodel can be further refined. Three sub-models of the manhole nozzle of the surge tank are shown in Fig 13 (the nozzle is marked by a square of dashed lines in the Fig. 12). The sub-model 2 and sub-model 3 are refinements (nested submodels) of the initial sub-model 1 .

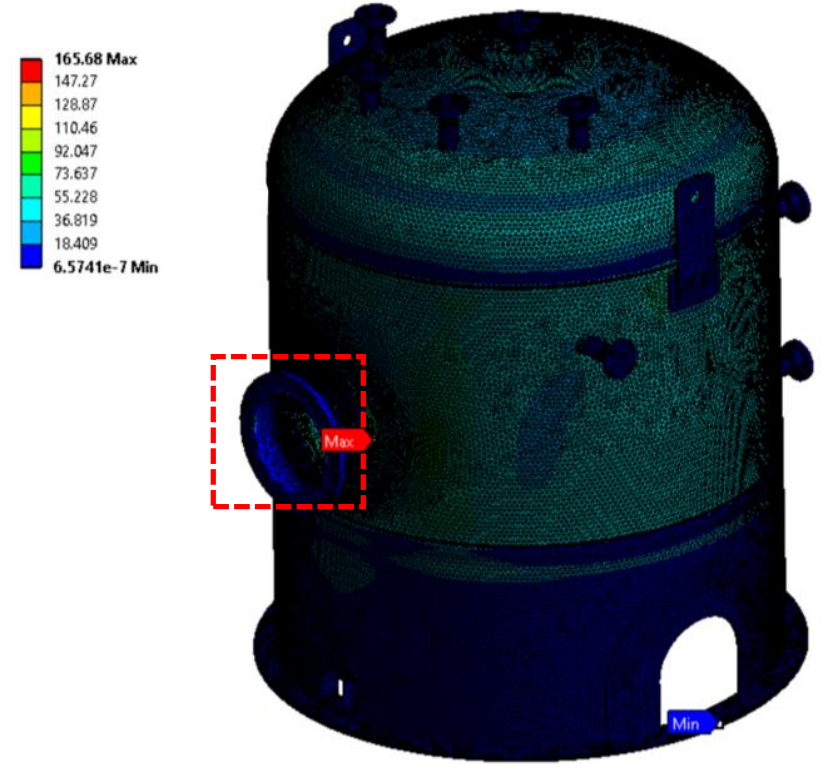

Fig. 12 Surge tank finite element model with the equivalent stress results and region of sub-modeling

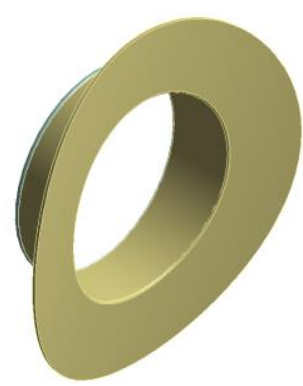

a

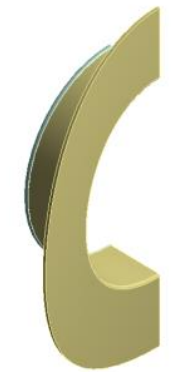

b

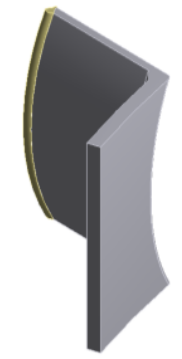

c
Fig. 13 Nested sub-models: a - sub-model 1 (an area of interest which is cut from global model), b - submodel 2 (sub-model of the sub-model 1) c - submodel 3 (sub-model of the sub-model 2)

The maximum equivalent stress calculated using the final sub-model 3 is $283 \mathrm{MPa}$ at the weld toe (Fig. 14). It is in 1.7 times large that the maximum stress in the global model. This technique allowed to calculate the maximum stress at the concentration zone, without a large number of finite elements and computational efforts using 47893 hex- ahedral shape solid elements dominant mesh with $3 \mathrm{~mm}$ average element size, comparing to 419797 solid elements of the global model with $14 \mathrm{~mm}$ average element size in the part of interest.

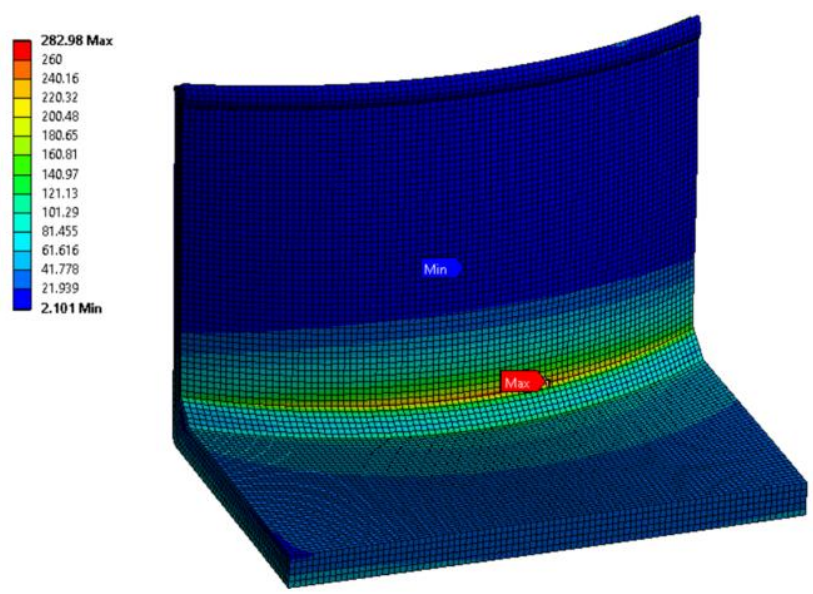

Fig. 14 Equivalent stresses at the refined sub-model 3

\section{Conclusions}

The displacement based sub-modelling results are less sensitive to the mesh density of the global model, comparing with the force based sub-modelling. However, force based sub-models can be used and give satisfactory results under moderate global model mesh density.

The displacement based sub-modelling cannot be applied without pre-examination in case if geometry of submodeled region is changed and the stiffness of the submodel is changed consequently. Force based sub-modelling should be preferred in that case.

The nested sub-modelling technique can be applied with the several refinements of the initial sub-model, when initially it is difficult so select the sub-model size and geometry from the global model.

\section{References}

1. Narvydas, E.; Puodziuniene, N. 2014. Applications of sub-modelling in structural mechanics, Proceedings of 19th International Conference Mechanika 2014, Kaunas, Lithuania, p. 172-176.

2. Wang, H.; Li. A.Q.; Guo, T.; Ma., S. 2009. Accurate stress analysis on rigid central buckle of long-span suspension bridges based on submodel method, Science in China Series E: Technological Sciences 52: 1019-1026. https://doi.org/10.1007/s11431-009-0070-z.

3. Liu Y., Stratman B., Mahadevan S. 2006. Fatigue crack initiation life prediction of railroad wheels, International Journal of Fatigue 28(7): 747-756. https://doi.org/10.1016/j.ijfatigue.2005.09.007.

4. Cormier, N.G.; Smallwood, B.S.; Sinclair, G. B.; Meda G. 1999. Aggressive submodelling of stress concentrations, International Journal for Numerical Methods in Engineering 46: 889-909. https://doi.org/10.1002/(SICI)10970207(19991030)46:6<889: AID-NME699>3.0.CO;2-F.

5. Martinsson, J. 2004. Automatic 3d crack propagation in complex welded structures, 15th European Conference on Fracture ECF15 Advanced Fracture Mechanics for Life and Safety Assessments. Stockholm, Sweden, p. $1-9$. 
6. Perić, M., Seleš, K., Tonković, Z., \& Lovrenić-Jugović, M. 2019. Numerical simulation of welding distortions in large structures with a simplified engineering approach, Open Physics 17(1): 719-730. https://doi.org/10.1515/phys-2019-0076.

7. Perić, M.; Tonković, Z.; Maksimović, K.S.; Stamenković, D. 2019. Numerical analysis of residual stresses in a T-Joint fillet weld using a submodeling technique, FME Transactions 47(1): 183-189. https://doi.org/10.5937/fmet1901183P.

8. Gonzalez-Albuixech, V. F.; Qian, G.; Sharabi, M.; Niffenegger, M.; Niceno, B.; Lafferty, N. 2015. Comparison of PTS analyses of RPVs based on 3D-CFD and RELAP5, Nuclear Engineering and Design 291: 168178.

https://doi.org/10.1016/j.nucengdes.2015.05.025.

9. Gonzalez-Albuixech, V. F.; Qian, G.; Sharabi, M.; Niffenegger, M.; Niceno, B.; Lafferty, N. 2016. Coupled RELAP5, 3D CFD and FEM analysis of postulated cracks in RPVs subjected to PTS loading, Nuclear Engineering and Design 297: 111-122. https://doi.org/10.1016/j.nucengdes.2015.11.032.

10. Sung, S. J.; Pan, J.; Lam, P. S.; Scarth, D. A. 2017. Ductile fracture initiation near axial crack fronts in pressure tubes of hydrided irradiated $\mathrm{Zr}-2.5 \mathrm{Nb}$ materials with split circumferential hydrides, Engineering Fracture Mechanics 186: 1-20. https://doi.org/10.1016/j.engfracmech.2017.09.003.

11. Sung, S. J.; Pan, J.; Lam, P.S.; Scarth, D. A. 2017. "Ductile fracture initiation with consideration of strain concentration and stress triaxiality near crack fronts in compact tension specimens of hydrided irradiated $\mathrm{Zr}$ $2.5 \mathrm{Nb}$ materials with split circumferential hydrides, Engineering Fracture Mechanics 186: 208-241. https://doi.org/10.1016/j.engfracmech.2017.10.012.

12. Mora, D. F.; Niffenegger, M.; Qian, G. A.; Jaros, M.; Niceno, B. 2019. Modelling of reactor pressure vessel subjected to pressurized thermal shock using 3D-XFEM, Nuclear Engineering and Design 353: 13. https://doi.org/10.1016/j.nucengdes.2019.110237.

13. Azzuni, E.; Guzey, S. 2019. Brittle fracture failure assessment of API 12D tanks with a semicircular-topped rectangular cleanout and stepped shell design, International Journal of Pressure Vessels and Piping 175:14. https://doi.org/10.1016/j.ijpvp.2019.05.011.

14. Danielczyk P. 2019 A Sub-modeling approach for building numerically efficient discrete model for shape optimization - a case study, In: Rusiński E., Pietrusiak D. (eds) Proceedings of the 14th International Scientific Conference: Computer Aided Engineering, CAE 2018. Lecture Notes in Mechanical Engineering. Cham: Springer, p. 120-127. https://doi.org/10.1007/978-3-030-04975-1_15.

15. Liu, Z.; Correia, J.; Carvalho, H.; Mourao, A.; de Jesus, A.; Calcada, R.; Berto, F. 2019. Global-local fatigue assessment of an ancient riveted metallic bridge based on submodelling of the critical detail, Fatigue $\&$
Fracture of Engineering Materials \& Structures 42(2): 546-560.

https://doi.org/10.1111/ffe.12930.

16. Pilthammar, J.; Sigvant, M.; Kao-Walte, S. 2018. Introduction of elastic die deformations in sheet metal forming simulations, International Journal of Solids and Structures 151: 76-90.

https://doi.org/10.1016/j.ijsolstr.2017.05.009.

17. Curreli, C.; Di Puccio, F.; Mattei, L. 2018. Application of the finite element submodeling technique in a single point contact and wear problem, International Journal for Numerical Methods in Engineering 116 (10-11): 708-722. https://doi.org/10.1002/nme.5940.

18. Shankar, S.; Nithyaprakash, R.; Santhosh, B. R.; Uddin, M. S.; Pramanik, A. 2020. Finite element submodeling technique to analyze the contact pressure and wear of hard bearing couples in hip prosthesis, Computer Methods in Biomechanics and Biomedical Engineering: 10. https://doi.org/10.1080/10255842.2020.1734794.

19. Abaqus 2016 Analysis User's Guide. 2015. v. II: Analysis, Dassault Systèmes, 1529 p.

20. ANSYS Help System. Advanced Analysis Guide. 2012. SAS IP, Inc.

21. SOLIDWORKS 2018. Online Help System. Dassault Systèmes, SOLIDWORKS Corporation.

22. Pilkey, W. D. 1997. Peterson's Stress Concentration Factors, 2nd ed. New York: John Wiley \& Sons, Inc. 544 p.

23. Young, W. C.; Budynas, R. G. 2002. Roark's Formulas for Stress and Strain. 7th edition. New York: McGrawHill. 852 p.

E. Narvydas, N. Puodziuniene, A. K. Thorappa

APPLICATION OF FINITE ELEMENT SUBMODELING TECHNIQUES IN STRUCTURAL MECHANICS

S u m m a r y

Sub-modeling techniques implemented in commercial finite element codes were reviewed. New sub-modeling applications in structural mechanics stress analysis are presented. Sub-modeling methods based on displacement and force cut boundary application are compared. Advantages and disadvantages of these methods are discussed. The case of nested sub-models is demonstrated.

Keywords: sub-modeling, model reduction methods, finite element analysis, stress analysis.

Received April 30, 2020

Accepted December 07, 2021

This article is an Open Access article distributed under the terms and conditions of the Creative Commons Attribution 4.0 (CC BY 4.0) License (http://creativecommons.org/licenses/by/4.0/). 